\title{
Guía de práctica clínica para tratamiento de fibrilación auricular
}

\author{
Víctor Hugo Forero ${ }^{1}$
}

1 Especialista en Medicina Familiar Integral, Magister en Epidemiología Clínica. Docenteinvestigador, Fundación Universitaria Juan N. Corpas.

Correspondencia: Dr. Víctor Hugo Forero, victor.forero@juanncorpas.edu.co

Como citar: Forero VH. Guía de práctica clínica para tratamiento de fibrilación auricular. Cuarzo 2015; 21 (1): 39-53.

Recibido: 26 de febrero de 2015

Aceptado: 16 de mayo de 2015.

Publicado: 30 de junio de 2015.

Licencia creative commons (c) (i) $\Theta$

\section{Resumen}

El tratamiento de la fibrilación auricular impone retos en cada caso. Nuestro propósito es brindar recomendaciones para el médico en atención primaria. Las fuentes de información principales en la elaboración de la guía fueron estudios primarios y agregativos.

En general, el tratamiento de la fibrilación auricular requiere enfoque individualizado y es altamente recomendable aprovechar de manera pertinente las escalas clínicas disponibles para identificación de beneficios y riesgos según las alternativas terapéuticas.

Para la toma de deciciones hay dos aspectos calve: 1) determinar justificación para profilaxis antitrombótica, 2) evaluar la estrategia más apropiada: control de frecuencia - control de ritmo. Las recomendaciones estan expuestas en tablas y algoritmos que requieren ser utilizadas según el contexto y características individuales en cada caso.

PALABRAS CLAVE: fibrilación auricular, embolísmo, anticoagulación, sangrado, guía práctica clínica, atención primaria.

\section{Abstract \\ Atrial flbrillation - practical clinical guideline}

Since the management of atrial fibrillation may be difficult in the individual patient, our purpose was to develop simple clinical recommendations to help the primary primary care physicians . SOURCES: Systematic review of the literature with evaluation of data-related evidence and framing of graded recommendations. SYNTHESIS: The management of atrial fibrillation requires individualised evaluation of the risks and benefits of therapeutic modalities, relying whenever possible on simple and validated tools. The two main points requiring a decision in clinical management are 1) whether or not to implement thromboembolic prevention therapy, and 2) whether preference should be given to a "rate control" or "rhythm control" strategy. To assist the physician in making recommendations for the care of an atrial fibrillation patient we propose specific tables and algorithms, with graded recommendations. CONCLUSIONS: On the evidence of data from the literature we propose simple algorithms and tables for the clinical management of atrial fibrillation in the individual patient.

KEYWORDS: guide clinical practice, atrial fibrillation, embolism, anticoagulation, bleeding, primary care. 


\section{Metodología}

\section{Grupo de trabajo en la elaboración de la guía de práctica clínica}

Especialistas en Medicina Familiar, Especialista en Medicina Interna

\section{Población usuaria de la guía práctica clínica}

Médicos generales, especialistas en Medicina Familiar, Medicina Interna, Cardiología

\section{Población objeto de la guía práctica clínica}

Pacientes adultos, de ambos sexos con diagnóstico de fibrilación auricular

\section{Objeto de la guía práctica clínica}

Brindar recomendaciones para el tratamiento de fibrilación auricular paroxística, persistente o permanente.

Para los efectos de la presente guía ésta es la definición operativa de fibrilación auricular: trastorno del ritmo cardíaco, taquicardia supraventricular, con activación auricular no coordinada, asociada o no a disfunción contráctil.

\section{Obtención de la evidencia}

La información y evidencia relevante se obtuvo de: Pubmed y Embase: con la siguiente sintaxis : (atrial fi- brillation and MANAGEMENT AND ( ( Review[ptyp] OR Meta-Analysis[ptyp] OR Clinical Trial, Phase III[ptyp] OR Clinical Trial, Phase IV[ptyp] ) AND "last 5 years"[PDat] AND adult[MeSH])) Filters: Review; Meta-Analysis; Clinical Trial, Phase III; Clinical Trial, Phase IV; published in the last 5 years; Adult: 19+ (a 31 de marzo 2015), lo cual generó 145 artículos potencialmente relevantes. Luego de analizar los abstracts y por selección de tipos de artículos específicos a los propósitos de la guía, resultaron 52 documentos utilizados para la construcción de las recomendaciones.

Las recomendaciones consignadas en esta guía fueron acordadas en consenso, análisis y discusión entre los profesionales y especialistas involucrados. La calidad y fortaleza de la evidencia se definió con base en lo expuesto en la tabla 1 .

\section{Proceso de elaboración de la guía}

- Previa revisión de la literatura seleccionada y apoyados en la experiencia clínica y evidencia científica aportada por los expertos se realizó un documento de trabajo.

- Se realizaron reuniones para presentación y discusión del documento de trabajo.

- De estas reuniones se recogieron los aportes y fueron realizados ajustes pertinentes al documento para versión final.

TABLA 1. Evaluación y selección de la información para las recomendaciones.

\section{Niveles de evidencia}

$\begin{array}{ll}\text { Nivel I } & \text { Evidencia lograda a partir de estudios controlados, aleatorio, bien diseñado. } \\ \text { Nivel II } & \text { - Estudios apropiadamente diseñados sin aleatorización } \\ & \text { - Estudios de cohorte o estudios analíticos de casos controlados } \\ & \text { - Análisis de series de casos con o sin intervención } \\ \text { Nivel III } & \text { - Estudios descriptivos } \\ & - \text { Reporte de casos } \\ & - \text { Informes de comités de expertos / organizaciones (por ejemplo, consenso del panel de especialistas) }\end{array}$

Grados de recomendación (fortaleza de evidencia)

Relevante per no crítico

Puntos clave Recomendaciones basadas en experiencia clínica y por consenso entre los desarrolladores de la guía de práctica clínica

Fuente: Autor. 


\section{Financiación}

Esta Guía de Práctica Clínica ha sido elaborada sin financiación externa o aportes diferentes al trabajo individual y de grupo que cada participante ha otorgado para su elaboración y a lo dispuesto por la Clínica Juan N. Corpas en tiempo contractual de algunos de los participantes, recursos instrumentales, y logísticos para su desarrollo, difusión e implementación.

\section{Declaración de conflictos}

En ninguna de las etapas de la producción de esta guía la Clínica Juan N. Corpas; ni los participantes en su elaboración, han suscrito o contraído compromiso o conflicto de interés alguno con agentes o entidades relacionadas con industria productora, distribuidora o representante de los insumos para la realización de pruebas de apoyo diagnóstico, ni farmacéutica o de elementos que apoyan las intervenciones terapéuticas recomendadas en esta guía.

\section{Recomendaciones}

Asegurar, como mínimo, los siguientes tres elementos

\section{A. Identificar en forma integral:}

- Compromiso vital

- Tipo de fibrilación auricular y posibles causas

\section{B. Intervención terapéutica}

- Triple objetivo [Recuperar- mantener función hemodinámica, prevenir tromboembolismo (proteger cerebro), alivio de síntomas]

\section{Asegurar plan de seguimiento y control.}

- Recurrencia

- Complicaciones

\section{A. Identificar en forma integral}

En todo paciente con presencia o manifestación de: disnea, palpitaciones, mareo, dolor torácico, evento o deterioro neurológico mediante evaluación mental abreviada (I-A) (1-3)

- Compromiso vital: estado hemodinámico, síntomas, signos y evaluación mental Identificar tensión arterial, pulso arterial junto con auscultación cardiaca,

- Factores de riesgo / precipitantes para FA (Edad avanzada, hipertensión, valvulopatía, cardiopatía, causas metabólicas, medicamentos, alcohol, tabaco, infecciosa)

- Determinar tipo de fibrilación auricular y posibles causas (cardiacas - no cardiacas)
Solicitar ECG. (II-B) (4-6)

Ante sospecha FA paroxística aún sin hallazgos positivos en ECG: se recomienda monitorización electrocardiográfica ambulatoria 24 horas

Solicitar Ecocardiograma transtorácico (II-A) (7-9):

- En paciente con fibrilación auricular y sospecha de enfermedad cardiaca estructural / funcional

- Cuando se requiere realizar cardioversión

Tipo de fibrilación auricular

- Aguda: episodio recurrente o nuevo de duración < 48 horas

- Paroxística: episodio recurrente o nuevo de duración $<7$ días y se resuelve espontáneamente

- Persistente: episodio recurrente o nuevo de duración $>7$ días y no resuelve espontáneamente

- Persistente de larga data: más de 1 año.

- Permanente: Se ha convenido este término para referirse a FA en la que se desiste de intervenir para restablecer a ritmo sinusal

- No valvular: FA sin existencia de valvulopatía reumática, reparación o prótesis valvular mitral.,

\section{B. Intervención terapéutica -Triple objetivo-}

- Recuperar y mantener estabilidad hemodinámica

- Prevenir complicaciones, especialmente eventos vasculares neurológicos.

- Aliviar los síntomas.

Recuperar y mantener estabilidad hemodinámica

En inestabilidad hemodinámica se recomienda realizar cardioversión eléctrica (I-B) (10).

Control frecuencia y ritmo.

Prevenir complicaciones, especialmente eventos vasculares neurológicos. PROTEGER CEREBRO.

Identificar riesgo de evento vascular: Escala CHADS2 \& CHA2DS2-VASc (I-B) (11-14)

- Pertinencia de anticoagulación y posibles efectos no deseados

Junto con la evaluación de riesgo de evento vascular se recomienda utilizar la escala HAS- BLED para evaluar el riesgo de sangrado en terapia anticoagulante (II-A) (19-21) 
Medicamentos y dosis para control de frecuencia cardíaca.

\section{Medicamento}

Metoprolol

2.5-5.0 $\mathrm{mg}$ en 2 minutos (hasta 3 dosis)

Propranolol

Esmolol

Verapamilo

$\mathrm{Cr}$

Diltiazem

Digoxina

Amiodarona
$1 \mathrm{mg}$ en 1 min (hasta 3 dosis con intervalo de 2 minutos)

$500 \mathrm{mcg} / \mathrm{kg}$ en carga durante 1 minuto luego $50-300 \mathrm{mcg} / \mathrm{kg} / \mathrm{min}$

$0.075-0.15 \mathrm{mg} / \mathrm{kg}$ en carga durante 2 minutos

Puede administrarse dosis adicional $10 \mathrm{mg}$ luego de 30 minutos

Continuar $0.005 \mathrm{mg} / \mathrm{kg} / \mathrm{min}$

$0.25 \mathrm{mg} / \mathrm{kg}$ carga durante 2 minutos, luego $5-15 \mathrm{mg} / \mathrm{h}$

$0.25 \mathrm{mg}$ (repetir dosis, de ser necesario, hasta $1.5 \mathrm{mg}$ en $24 \mathrm{~h}$ )

5-7 $\mathrm{mg} / \mathrm{kg}$ durante los primeros 60 minutos luego $10-50 \mathrm{mg} / \mathrm{h}$ por las siguientes 24 horas
25-100 mg (dos veces día)

10-40 mg (3-4 veces día)

180-480 mg (una vez día)

120-360 mg (una vez día)

0.125-0.25 mg (una vez día)

100-200 mg (una vez día)

La selección del medicamento se realiza conbase en las características de cada caso.

Variables y escala para valoración de riesgo de evento vascular (CHADS2 \& CHA2DS2-VASc).

\begin{tabular}{ll}
\hline Insuficiencia cadíaca congestiva/disfunción ventricular izquierda* & 1 \\
Hipertensión* & 1 \\
\hline Edad $\geq 75$ & 2 \\
\hline Diabetes mellitus* & 1 \\
ACV / ataque isquémico transitorio / tromboembolismo & 2 \\
Enfermedad vascular & 1 \\
Edad 65-74 & 1 \\
Mujer & 1 \\
\hline
\end{tabular}

CHADS2 o CHA2DS2-VASc igual o mayor de 2 se recomienda anticoagulación oral (salvo que exista contraindicación) bien con antagonistas de vitamina K o con anticoagulantes orales directos (Apixaban, Dabigatran, Rivaroxaban) (I-A,II-B) (15-18).

*Cada uno de los factores contribuyentes y modificables a evento vascular embolico requiere intervención.

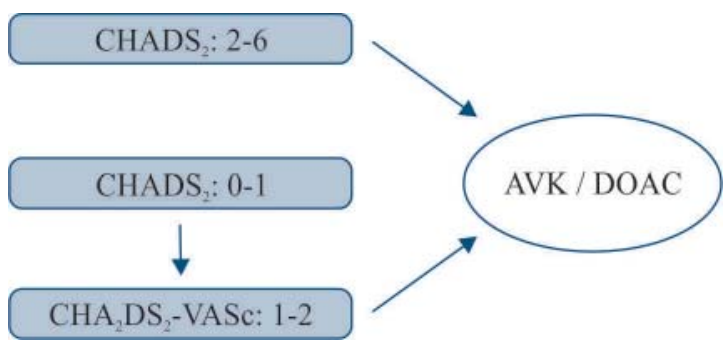

$\mathrm{AVK}=$ Antagonista de vitamina $\mathrm{K}-\mathrm{DOAC}=$ Anticoagulante oral directo.

Opciones de anticoagulante oral según riesgo de evento vascular 
La selección de terapia anticoagulante requiere ser realizada con base en las necesidades, preferencias, circunstancias y especificidad en cada caso. Para orientar la selección de la terapia anticoagulante es indispensable atender las características de las diferentes opciones.

Escala HAS- BLED para evaluar el riesgo de sangrado en terapia anticoagulante.

\begin{tabular}{lll}
\hline H & Hipertensión & 1 \\
A & Alteración hepática o renal (abnormal) & 1 (por cada alteración) \\
S & Evento vascular cerebral (stroke) & 1 \\
B & Hemorragia (Bleeding) & 1 \\
L & INR inestable (Lability) & 1 \\
E & Edad avanzada (elderly) & 1 \\
D & Drogas/alcohol (drugs) & 1 (por cada item) \\
\hline
\end{tabular}

0-2: riesgo de sangrado bajo-moderado

$3 \geq$ : riesgo de sangrado alto

Características farmacológicas de de anticoagulante oral.

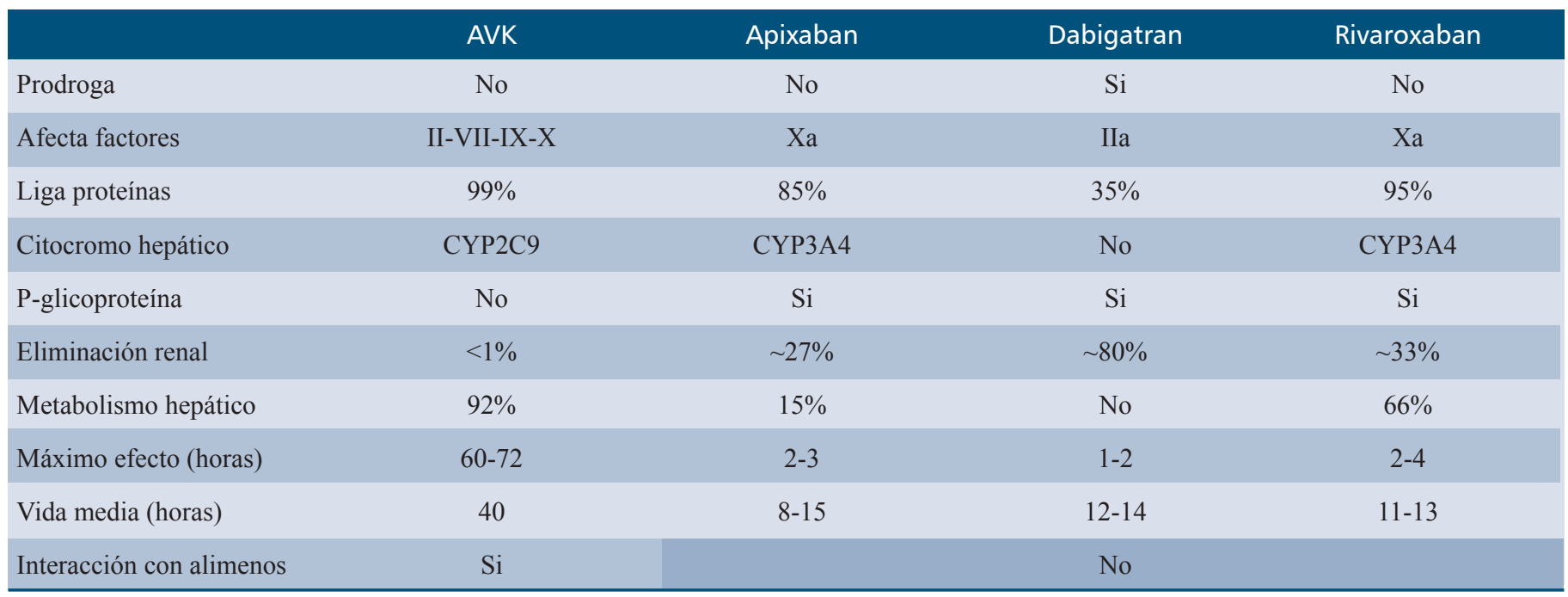

\section{Asegurar plan de seguimiento y control.} Identificar y comunicar al paciente y familia sobre:

- Riesgo de evento vascular

- Pertinencia de anticoagulación y posibles efectos no deseados

La elección de terapia antitrombótica se realiza con base en el riesgo de embolismo independientemente del tipo de FA (I -B) (22-24)
Sea cual sea la opción anticoagulante recomendada, asegurar:

- Comprensión clara por parte del paciente y su familia.

- Reconciliación de medicamentos.

- Anticipación de los riesgos e identificación de efectos secundarios.

- Orientación sobre conducta a seguir ante dudas o presencia de eventos secundarios. 
FIBRILACIÓN AURICULAR

(Disnea, palpitaciones, mareo, color torácico, evento o deterioro neurológico)

$1^{\circ}$ IDENTIFICAR RIESGO VITAL

Estado hemodinámico, síntomas, signos y evaluación mental

$2^{\circ}$ IDENTIFICAR FACTORES DE RIESGO

Características generales

Edad

Peso

Raza

Tabaquismo

Uso de psicoactivos

Condición clínica
Hipertensión
Diabetes
Cardiopatia
Trastorno metabólico

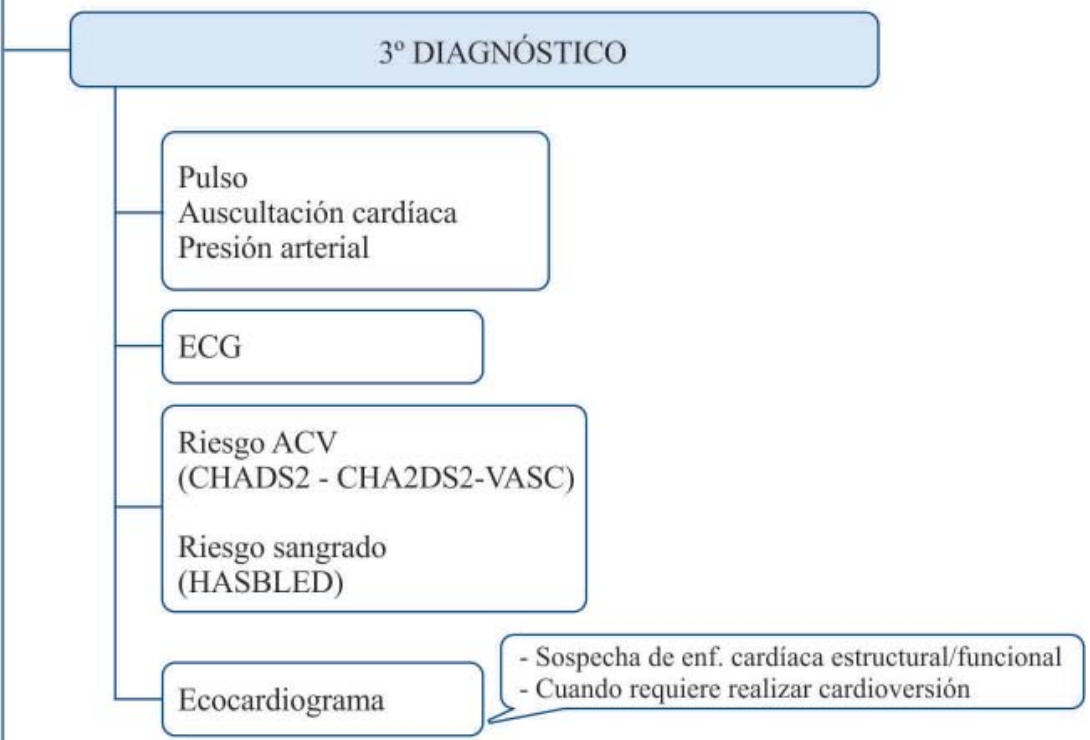

\section{$4^{\circ}$ DETERMINAR TRATAMIENTO}

- Recuperar y mantener estabilidad hemodinámica

- Aliviar síntomas

- Prevenir complicaciones (ACV)

Farmacológica

No farmacológica

$5^{\circ}$ PLAN DE EVALUACIÓN Y SEGUIMIENTO 


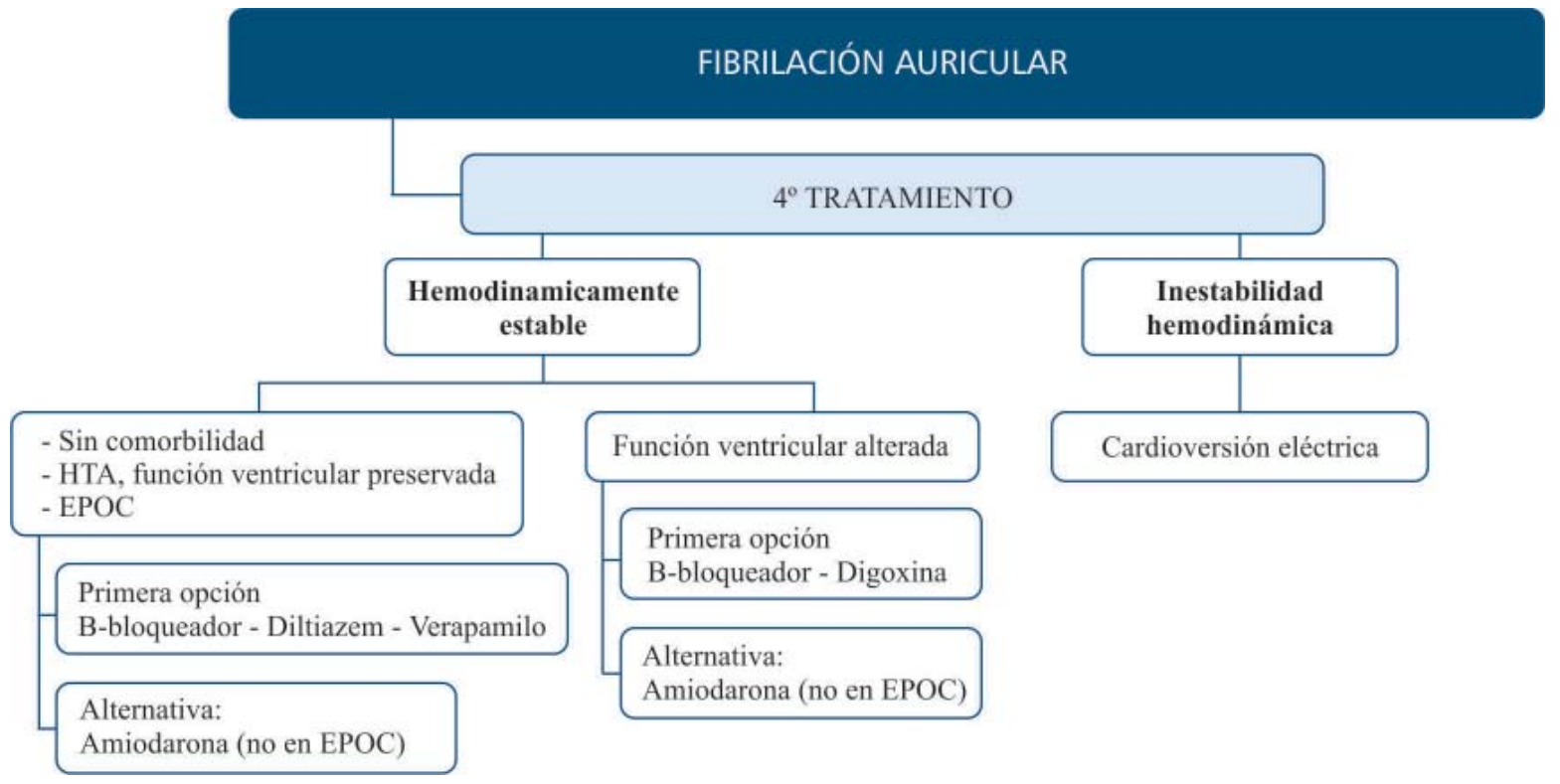

Medicamentos y dosis para control de frecuencia cardíaca.

\section{Medicamento}

Dosis IV

Dosis oral

\begin{tabular}{|c|c|c|}
\hline Metoprolol & 2.5-5.0 mg en 2 minutos (hasta 3 dosis) & 25-100 mg (dos veces día) \\
\hline Propranolol & $1 \mathrm{mg}$ en $1 \mathrm{~min}$ (hasta 3 dosis con intervalo de 2 minutos) & 10-40 mg (3-4 veces día) \\
\hline Esmolol & $\begin{array}{l}500 \mathrm{mcg} / \mathrm{kg} \text { en carga durante } 1 \text { minuto } \\
\text { luego } 50-300 \mathrm{mcg} / \mathrm{kg} / \mathrm{min}\end{array}$ & \\
\hline Verapamilo & $\begin{array}{l}0.075-0.15 \mathrm{mg} / \mathrm{kg} \text { en carga durante } 2 \text { minutos } \\
\text { Puede administrarse dosis adicional } 10 \mathrm{mg} \text { luego de } 30 \text { minutos } \\
\text { Continuar } 0.005 \mathrm{mg} / \mathrm{kg} / \mathrm{min}\end{array}$ & 180-480 mg (una vez día) \\
\hline Diltiazem & $\begin{array}{l}0.25 \mathrm{mg} / \mathrm{kg} \text { carga durante } 2 \text { minutos } \\
\text { luego } 5-15 \mathrm{mg} / \mathrm{h}\end{array}$ & 120-360 mg (una vez día) \\
\hline Digoxina & $0.25 \mathrm{mg}$ (repetir dosis, de ser necesario, hasta $1.5 \mathrm{mg}$ en $24 \mathrm{~h}$ ) & 0.125-0.25 mg (una vez día) \\
\hline Amiodarona & $\begin{array}{l}5-7 \mathrm{mg} / \mathrm{kg} \text { durante los primeros } 60 \text { minutos } \\
\text { luego } 10-50 \mathrm{mg} / \mathrm{h} \text { por las siguientes } 24 \text { horas }\end{array}$ & 100-200 mg (una vez día) \\
\hline
\end{tabular}

La selección del medicamento se realiza conbase en las características de cada caso.

Medicamentos y dosis para control de ritmo.

\section{Medicamento}

Flecainida

Propafenona

Amiodarona
Dosis IV

$1.5-3 \mathrm{mg} / \mathrm{kg}$ en $10-20$ minutos

$5-7 \mathrm{mg} / \mathrm{kg}$ durante los primeros 60 minutos luego $10-50 \mathrm{mg} / \mathrm{h}$ por las siguientes 24 horas

5-7 mg/kg durante los primeros 60 minutos luego $10-50 \mathrm{mg} / \mathrm{h}$ por las siguientes 24 horas

\section{Dosis oral}

200-300 mg (una vez día)

100-200 mg (una vez día)

100-200 mg (una vez día) 
- Anticipación de los riesgos e identificación de efectos secundarios

- Orientación sobre conducta a seguir ante dudas o presencia de

eventos secundarios

\section{Puntos clave}

- FA puede ser asintomática hasta en $50 \%$ de las personas jóvenes. En $20 \%$ de la población general cursa sin clínica evidente.

- Aunque la FA puede ser asintomática por largo tiempo, el impacto funcional, hemodinámico y trombogénico es un hecho!.

- La fibrilación auricular aumenta el riesgo de accidente cerebrovascular en aproximadamente 5 veces.

- Uno de cada cinco ACV está asociado a FA.

- Riesgo embólico (CHADS2) $\geq 2$ se recomienda anticoagulación oral, independiente del tipo de FA.

- No hay evidencia de que el riesgo de tromboembolismo o accidente cerebrovascular difiera entre los métodos de cardioversión farmacológica o eléctrica por lo tanto, la recomendación anticoagulación aplica para las dos estrategias.

- La elección de terapia antitrombótica se acompaña de evaluación continuada de riesgo de sangrado.

- Alerta. En síndromes de pre-excitación no administrar fármacos que "enlentecen" el nodo AV.

Paciente con FA:

- Mantener, recuperar estabilidad hemodinámica
- Reducir riesgo de evento tromboembólico [proteger cerebro]

- Aliviar síntomas

El control de la frecuencia es la recomendación de primera línea para todos los pacientes, especialmente en:

- $\quad$ Edad $\geq 65$ años.

- $\quad$ FA cuyo inicio sea $\geq 48$ horas o cuando no se conoce la duración

- FA permanente

- FA asociada a enfermedad coronaria

- Cuando hay contraindicaciones a fármacos antiarrítmicos.

- Candidatos no apropiados para cardioversión (lesión estructural, intentos fallidos)

El control del ritmo está recomendado en:

- Paciente joven con FA sintomática

- FA aguda, primera vez, aislada

Siempre realizar reconciliación con otros medicamentos

Las interacciones entre medicamentos pueden precipitar o mantener la FA y otras taquiarritmias. 


\section{Anexos}

Fibrilación auricular - Auricular fibrillation: definición (MeSH): "Abnormal cardiac rhythm that is characterized by rapid, uncoordinated firing of electrical impulses in the upper chambers of the heart (HEART ATRIA). In such case, blood cannot be effectively pumped into the lower chambers of the heart (HEART VENTRICLES). It is caused by abnormal impulse generation".

¿Cuán frecuente es la fibrilación auricular?

La fibrilación auricular (FA) es el trastorno del ritmo cardiaco más común en el mundo, con gran impacto en la salud pública debido al aumento del riesgo de evento vascular cerebral y hospitalizaciones asociadas. Los resultados publicados sobre la epidemiología de la fibrilación auricular de la Carga Global de Enfermedades, traumatismos y factores de riesgo confirman un aumento mundial significativo y progresivo en la carga de la FA, para 2010 se calculó que el $0.5 \%$ de la población mundial (33.5 millones de personas) convive con FA. La prevalencia aumenta a medida que se ganan años: $1.5 \%$ hacia los 60 y $9.5 \%$ a los 75 y más años (25).

¿Cuál es el impacto en salud individual?

Para la población en Sur América la FA causa muerte a $1.20 / 100.000$ mujeres $(0.93-1.61)$ y $1.40 / 100.000$ hombres $(0.96$ - 2.24) , razón de mortalidad mujer: hombre $=0.86$.

¿Qué factores de riesgo subyacen a FA?

Después de la sexta década de la vida, la prevalencia de FA se duplica aproximadamente cada 10 años, de 0,5 $\%$ entre 50 -59 años a casi el $9 \%$ entre los 80 años a 89 años.

La FA es una condición compleja y multifactorial. En uno de los modelos predictivos más interesantes se han establecido como los principales: edad mayor a 60 años, sobrepeso, hipertensión arterial, fumar, raza negra, uso de medicación antihipertensiva, diabetes, y la historia de infarto de miocardio e insuficiencia cardíaca. En conjunto permiten establecer una probabilidad de FA de 0.76 (IC $95 \% 0.74-0.78)(26)$.

¿Cuáles son las formas de presentación?

El esquema de clasificación acordado entre sociedades cardiovasculares clasifica la FA según el patrón temporal de presentación y la presencia o no de enfermedad valvular:

- Aguda: episodio recurrente o nuevo de duración $<48$ horas

- Paroxística: episodio recurrente o nuevo de duración $<7$ días y se resuelve espontáneamente

- Persistente: episodio recurrente o nuevo de duración $>7$ días y no resuelve espontáneamente

- Persistente de larga data: más de 1 año.

- Permanente: Se ha convenido este término para referirse a FA en la que se desiste de intervenir para restablecer a ritmo sinusa

FA no valvular: sin existencia de valvulopatía reumática, reparación o prótesis valvular mitral.

Es necesario tener presente que esta forma de clasificar el tipo de FA está construida con sentido clínico práctico, no es exhaustiva, ni sus categorías son mutuamente excluyentes. Ejemplo, en un paciente podría identificarse en un momento dado FA paroxística pero tener características de FA persistente.

¿Cómo es el cuadro clínico?

Síntomas

Puede ser asintomática hasta en el 50\% de las personas jóvenes y en el $20 \%$ de la población general cursa sin clínica evidente.

Puede manifestarse como fatiga, palpitaciones y, al contrario de la taquicardia sinusal, su inicio y terminación suelen ser súbitos.

Si se acompaña de angina la probabilidad de enfermedad coronaria subyacente es elevada.

Si se acompaña de disnea suele existir condición mórbida pulmonar.

Los episodios de exacerbación suelen presentarse en personas con insuficiencia cardiaca y están relacionados con la disminución del gasto cardíaco.

Signos

El pulso es irregular, el primer ruido cardiaco puede tener amplitud variable. La diferencia entre la frecuen- 
cia de pulso radial y la frecuencia del impulso cardíaco identificada con el fonendoscopio (costumbre sana, tristemente no siempre practicada en la actualidad) es lo que se conoce como pulso deficitario o diferencial. Es el hallazgo más común en FA.

En la fibrilación auricular, el intervalo entre latidos ventriculares es al azar y es característico identificar pausas de variable duración. Por el contrario, en contracciones ventriculares prematuras, esto no sucede. Esta diferencia en el ritmo del pulso y del impulso cardiaco, que de nuevo se identifica con la sana costumbre de tomar el pulso y en simultáneo auscultar el corazón, se hace evidente con la práctica de las "sanas costumbres".

La exploración física permite en general establecer el diagnóstico, ya que el pulso arterial es irregular y rápido, excepto cuando el paciente ha recibido tratamiento. El primer ruido cardíaco varía de intensidad constantemente y en el pulso yugular se comprueba la ausencia de ondas a.

¿Cuándo y por qué realizar ECG?

En quien, con o sin síntomas presenta irregularidad de ritmo cardiaco. Es necesario para identificar el tipo de arritmia.

Detectar FA en el ECG, en el contexto de disfunción ventricular izquierda, representa sensibilidad de $26 \%$, especificidad de 93\%, LR+ 3.8 (IC95\%1.7-8.8), LR- 0.79 (IC95\% 0.65-0.96)

¿Hallazgos relacionados con sobrecarga o hipertrofia ventricular izquierda?

¿Hallazgos relacionados con: enfermedad coronaria?

¿Hallazgos relacionados con: trastornos en la conducción? (preexitación, bloqueos, repolarización)

En las personas con sospecha de fibrilación auricular paroxística y sin hallazgos positivos en ECG: se recomienda monitorización electrocardiográfica, ambulatoria, 24 horas (4-6).

¿Cuándo y por qué realizar Ecocardiograma en alguien con FA?

- Cuando se está considerando una estrategia de control del ritmo que incluye la cardioversión (eléctrica o farmacológica )
- Cuando hay alto riesgo o sospecha de enfermedad de base estructural / funcional cardíaca y que influye para recomendar el tipo de tratamiento

- Cuando sea necesario en la evaluación del riesgo clínico para el tratamiento antitrombótico (7-9)

No se justifica la solicitud rutinaria de ecocardiografía con el único propósito de la estratificación del riesgo de accidentes cerebrovasculares en personas con fibrilación auricular para quienes la necesidad de iniciar tratamiento anticoagulante ya se ha acordado con criterios clínicos apropiados.

¿Qué debe lograr el tratamiento de FA?

Hay tres elementos clave al plantear el tratamiento de FA:

- Recuperar y mantener estabilidad hemodinámica

- Aliviar los síntomas.

- Prevenir complicaciones, especialmente eventos vasculares neurológicos.

Aunque la FA puede ser asintomática durante largos períodos de tiempo, el impacto funcional, hemodinámico y trombogénico es un hecho y vale destacar:

1. La pérdida de la contribución auricular a la contracción cardíaca predispone y profundiza la insuficiencia cardíaca,

2. La asincronía y falla de contracción auricular facilita la formación de trombos

3. La pausa sinusal en el momento de cesar la crisis de fibrilación subyace a la presentación de síncope

4. Las palpitaciones son origen de ansiedad y seria molestia para el paciente (27-30).

¿Cómo recuperar y mantener estabilidad hemodinámica en FA... control de frecuencia o control de ritmo?

La estrategia de control de la frecuencia implica el uso de agentes betabloqueantes, antagonistas del calcio o digoxina, o una combinación de los mismos para controlar los síntomas al tiempo que permiten la "existencia" de FA. La estrategia de control del ritmo incluye cardioversión, ablación, o tratamiento con fármacos antiarrítmicos.

Este planteamiento ("frecuencia vs ritmo") es motivo de debate y siguen en curso estudios que comparan antiarrítmicos convencionales para el control del ritmo con fármacos para el control de la frecuencia. Teóricamente 
hay beneficios superiores en la restauración y el mantenimiento del ritmo, sin embargo la evidencia para afirmar ventajas sobre la reducción de la frecuencia no es concluyente; incluso existe información contradictoria.

Vale destacar cinco ensayos clínicos que comparan las dos estrategias de tratamiento: PIAF (Pharmacological Intervention in Atrial Fibrillation), STAF (Strategies of Treatment of Atrial Fibrillation), RACE (Rate Control versus Electrical conversion), AFFIRM (Atrial Fibrillation Followup Investigation of Rhythm Management) y HOTCAFE (How to Treat Chronic Atrial Fibrillation).

Los resultados de estos estudios indican que una estrategia de control de la frecuencia en los pacientes con FA puede ser al menos tan eficaz como los esfuerzos para controlar el ritmo. Sin embargo, estos ensayos tenían diferentes criterios de selección de pacientes y diferencia de criterios de valoración de los desenlaces, lo cual limita la aplicabilidad de sus resultados a todas las poblaciones con FA. Por otra parte, en estos mismos estudios se evidencia y reitera la necesidad de continuar el tratamiento antitrombótico, incluso cuando se obtiene el ritmo sinusal a largo plazo (31-35).

Ahora, útil resulta la revisión sistemática de Al- Khatib y colegas (10) que incluyó162 estudios y reúne 28.836 pacientes. Compara efectividad entre frecuencia versus ritmo. En términos de mortalidad por todas las causas OR 1,34 (IC $95 \%, 0,89-2.02$ ), mortalidad por causa cardiaca OR: 0,96 (IC95\% 0.77 a 1.20$)$ y accidente cerebrovascular OR 0,99 (IC95\% 0.76 a 1.30). Con estos datos, aunque la fuerza de la evidencia sea "modesta" está a favor de efectividad clínica superior para el control de la frecuencia. Lograr reducciones de frecuencia cardiaca en reposo $<110$ latidos por minuto es una recomendación razonable siempre y cuando no exista sintomatología limitante y la función sistólica esté preservada $(34,36,37)$.

Si bien el control de la frecuencia aplica a todos los pacientes, el control del ritmo es la recomendación de primera línea específicamente en pacientes con inestabilidad hemodinámica o en quienes permanecen clínicamente sintomáticos a pesar de suficiente control de la frecuencia $(38,39)$.

\section{¿Con qué opciones terapéuticas tratar FA?}

Los agentes farmacológicos incluyen: antiarrítmicos y anticoagulantes (reducir riesgo de accidente cerebrovascular).
Las opciones no farmacológicas incluyen: cardioversión eléctrica y catéter o ablación quirúrgica para suprimir los impulsos eléctricos anormales.

De nuevo, las opciones de intervención a FA se recomiendan, en cada caso, con base en el estado hemodinámico, comorbilidad, presencia o no de enfermedad valvular, tiempo de existencia de FA.

De existir inestabilidad hemodinámica la recomendación de primera línea es cardioversión eléctrica.

\section{Alerta con WPW}

Hay que mencionar de forma muy especial la FA en un paciente con síndrome de Wolff-Parkinson- White ("FA preexitada"). Se diagnostica al evidenciarse en el electrocardiograma, taquiarritmia de complejos anchos y de diferente morfología. Se tratará como una emer $\neg$ gencia y requiere cardioversión eléctrica sincronizada de urgencia. En estos casos están contraindicados los fármacos que "enlentecen" el nodo AV (digital, verapamilo, diltiazem, etc.).

La reducción de la frecuencia ventricular se puede lograr con beta bloqueador, o con antagonista de los canales de calcio, o con amiodarona siempre que no exista síndrome de preexitación. Se puede recomendar monoterapia con digoxina en casos de FA no paroxística, preferiblemente si se trata de personas sedentarias.

Cuando con monoterapia no se logra el efecto deseado, la combinación de digoxina y un bloqueador beta (o un antagonista de los canales de calcio) puede ser una buena alternativa en pacien tes con falla cardiaca sin alteración de la fracción de eyección $(36,40)$.

En pacientes jóvenes; sin cardiopatía asociada y que presentan frecuentes episodios de FA con pobre o no respuesta a las opciones farmacológicas, se puede realizar ablación de venas pulmonares como tratamiento de la FA.

¿Cómo enfrentar el riesgo de evento embólico cerebral?

Enfrentar los fenómenos tromboembólicos y el riesgo de sangrado cuando se decide por terapia anticoagulante no es asunto fácil y bien haremos en no desestimar los desafíos que involucran a pacientes, médicos y sistema de salud en general, para incorporar de manera juiciosa las alternativas anticoagulantes. 
Medicamentos y dosis para control de frecuencia cardíaca.

Medicamento

Dosis IV

Dosis oral

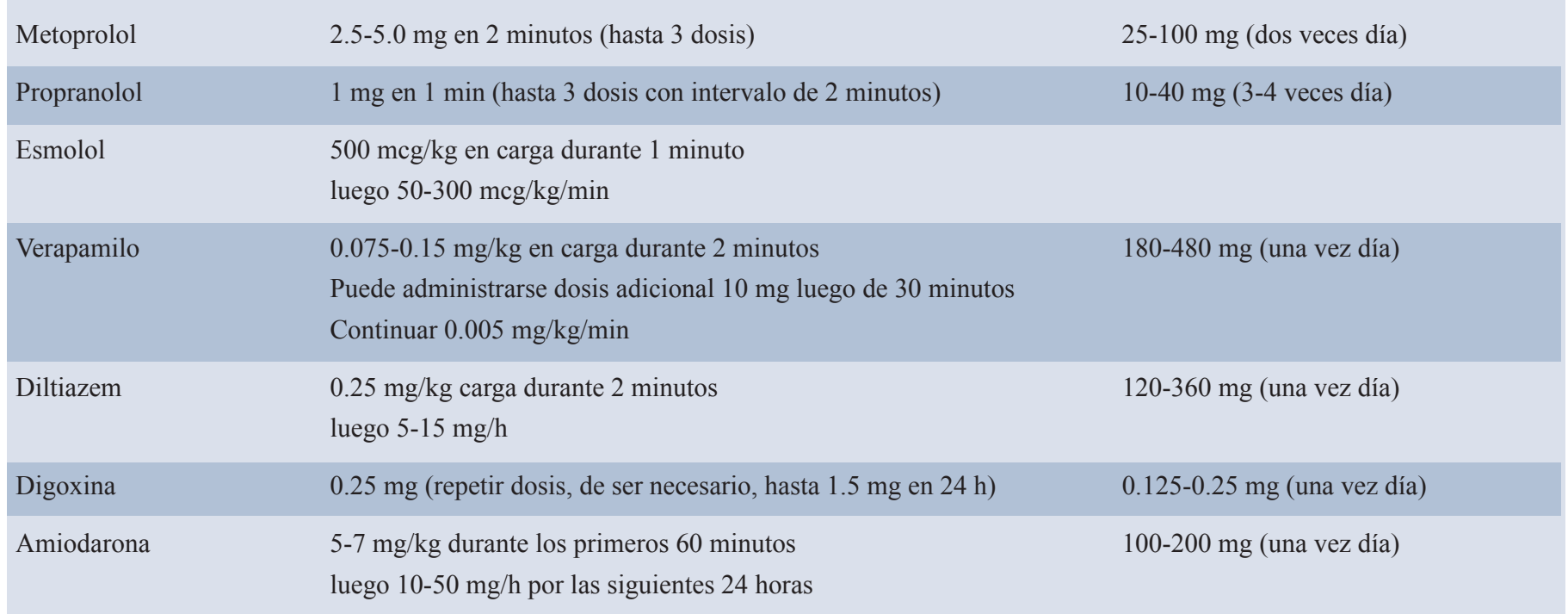

La selección del medicamento se realiza conbase en las características de cada caso.

Medicamentos y dosis para control de ritmo.

\section{Medicamento}

\section{Dosis IV}

\section{Dosis oral}

$\begin{array}{llr}\text { Flecainida } & 1.5-3 \mathrm{mg} / \mathrm{kg} \text { en 10-20 minutos } & \text { 200-300 mg (una vez día) } \\ \text { Propafenona } & \begin{array}{l}5-7 \mathrm{mg} / \mathrm{kg} \text { durante los primeros } 60 \text { minutos } \\ \text { luego } 10-50 \mathrm{mg} / \mathrm{h} \text { por las siguientes } 24 \text { horas }\end{array} & 100-200 \mathrm{mg} \text { (una vez día) } \\ \text { Amiodarona } & \begin{array}{l}5-7 \mathrm{mg} / \mathrm{kg} \text { durante los primeros } 60 \text { minutos } \\ \text { luego } 10-50 \mathrm{mg} / \mathrm{h} \text { por las siguientes } 24 \text { horas }\end{array}\end{array}$

Si la puntuación (CHADS) es igual o mayor de 2 se recomienda anticoagulación oral (salvo que exista contraindicación) bien con antagonistas de vitamina $\mathrm{K}$ o con anticoagulantes orales directos (Apixaban, Dabigatran, Rivaroxaban) (15-18) La puntuación HAS- BLED es una herramienta para guiar la decisión de iniciar la anticoagulación en pacientes con fibrilación auricular.

Con base en la información disponible en estudios aleatorizados con doble enmascaramiento, en pacientes con fibrilación auricular no valvular, se ha identificado que de los anticoagulantes directos: Rivaroxaban, Apixa- ban, (inhibidores de factor Xa), Dabigatran (inhibidor directo de trombina), se puede esperar eficacia semejante o superior a la que se ha obtenido con anticoagulantes AVK en prevención de ictus o embolia sistémica.

El meta-análisis de los ensayos clínicos identificó reducción en 19\% para el riesgo relativo de ictus o embolia sistémica en comparación con Warfarina (RR 0.81, IC 95\% 0.73-0.91; $\mathrm{p}<0.0001)$, también reducción de mortalidad por todas las causas (RR 0.90, IC 95\% $0.85-0.95$, $\mathrm{p}=0,0003)$ y de hemorragia intracraneal (RR 0.48, IC $95 \% 0.39-0.59, \mathrm{p}<0,0001)$, pero aumentó el riesgo de 
sangrado gastrointestinal (RR 1.25, IC 95\% $1.01-1.55$, p $=0,04)$. Lo que significa un riesgo $25 \%$ más alto con anticoagulación directa en comparación con AVK. (41-44).

¡Bien... el paciente recupera el ritmo sinusal luego de tratar la FA!...

¿Cómo continuar para mantener el ritmo sinusal y reducir el riesgo de recurrencia de FA?

Adicional al control de los factores predisponentes o asociados a recurrencia de FA es necesario el mantenimiento con alguno de los medicamentos disponibles, cuya elección y selección (asunto complejo) debe realizarse con base en la integración de múltiples factores en cada caso.

En el estudio de Freemantle (45) la amiodarona tuvo el mayor efecto en la reducción de recurrencia de FA (OR 0.22; IC del 95\%: 0,16 hasta 0.29), desafortunadamente se asoció con la mayor tasa de pacientes que experimentaron al menos un evento adverso grave: OR 2.41, IC95\% 0.96-6.0) adicional a los abandonos de tratamiento: OR 2.91, IC \% 1.66-5.11).

Dronedarona se asoció con la menor tasa de eventos pro-arrítmicos incluyendo bradicardia: OR 1.45 IC95\% 1.2 a 2.8), y con reducción significativa en el riesgo de ACV.OR 0.69 (IC95\% 0.57-0.84).

Sotalol registró las tendencias más altas a una mayor mortalidad: OR 3,44 ; (IC95\% 1.02 a 11.59), junto con amiodarona: OR 2.17 ; (IC95\% 0.63 a 7.51 )

Para corroborar los datos publicados por Freemantle, resulta clave y actualizada a 2015 la revisión sistemática de ensayos controlados aleatorios (actualización de la publicación en 2007) hecha por Lafuente- Lafuente y cols (46) que analiza 59 estudios con 21.305 pacientes quienes recuperaron el ritmo luego de tratar la FA. Este trabajo evidencia que los medicamentos clase IA (disopiramida, quinidina), IC (flecainida, propafenona) y III (amiodarona, dofetilida, dronedarona, sotalol) reducen significativamente la recurrencia de FA: OR 0.19 a 0.70 , (NNT 3 a 16).

Para el grupo de los beta-bloqueadores (específicamente metoprolol) la reducción de recurrencia FA (OR 0.62, (IC95\% 0.44-0.88), (NNT 9) Por otra parte el riesgo de muerte por todas las causas para los antiarrítmicos clase IA (quinidina y disopiramida) resulto significativa- mente mayor: OR 2.39; (IC 95\% 1.03 a 5.59) (NNT 109; IC95\%:34 a 4985) y para sotalol: OR 2.23; (IC95\%:1.1 a 4.50), (NNT 169; IC95\%: 60 a 2068). No se observó modificación en mortalidad con los otros grupos de antiarrítmicos

Así pues, con base en esta información, es razonable recomendar el tratamiento con agentes b-bloqueantes como la opción con el más apropiado beneficio clínico neto: efectividad con menor efecto no deseado, para reducir el riesgo de recurrencia de FA.

\section{Reconciliación medicamentos}

Las interacciones entre medicamentos pueden precipitar o mantener la FA y otras taquiarritmias.

Es clave realizar reconciliación de medicamentos: digoxina, simpaticomiméticos, antidepresivos tricíclicos, anticolinérgicos, tratamientos o principios activos de uso frecuente en migraña.

Algunas sustancias que puedan contener catecolaminas o compuestos semejantes a teofilina. Medicamentos que afectan canales iónicos, tales como terfenadina, cisaprida, astemizol.

\section{Referencias}

1. Steinberg B, Kim S, Thomas L, et al. Lack of Concordance Between Empirical Scores and Physician Assessments of Stroke and Bleeding Risk in Atrial Fibrillation: Results From the Outcomes Registry for Better Informed Treatment of Atrial Fibrillation (ORBIT-AF) Registry. Circulation. 2014;129(20):20052012.

2. Penado S, Cano M, Acha O, Hernández J, Riancho J. Atrial fibrillation as a risk factor for stroke recurrence. The American Journal of Medicine. 2003;114(3):206210.

3. Abraham JM, Connolly SJ. Atrial fibrillation in heart failure: stroke risk stratification and anticoagulation. Heart Fail Rev. 2014;19(3):305-13.

4. Taggar JS, Coleman T, Lewis S, Heneghan C, Jones M. Accuracy of methods for diagnosing atrial fibrillation using 12-lead ECG: A systematic review and meta-analysis. Int J Cardiol. 2015;184C:175-183.

5. Shi X-MM, Yuan H-TT, Guo H-YY, Guo J-PP, Shan Z-LL, Wang Y-TT. Electrophysiological characteristics of paroxysmal atrial fibrillation originating from superior vena cava: a clinical analysis of 30 cases. Int J Clin Exp Med. 2015;8(1):240-8.

6. Sposato LA, Cipriano LE, Saposnik G, Vargas ER, Riccio PM, Hachinski V. Diagnosis of atrial fibrillation after stroke and transient ischaemic attack: a systematic review and meta-analysis. Lancet Neurol. 2015;14(4):377-87. 
7. Luong C, Thompson DJ, Bennett M, et al. Right atrial volume is superior to left atrial volume for prediction of atrial fibrillation recurrence after direct current cardioversion. Can J Cardiol. 2015;31(1):29-35.

8. Yoon YE, Oh I-YY, Kim S-AA, et al. Echocardiographic Predictors of Progression to Persistent or Permanent Atrial Fibrillation in Patients with Paroxysmal Atrial Fibrillation (E6P Study). J Am Soc Echocardiogr. 2015.

9. Bekwelem W, Misialek JR, Konety S, et al. Echocardiographic measures of cardiac structure and function are associated with risk of atrial fibrillation in blacks: the Atherosclerosis Risk in Communities (ARIC) study. PLoS ONE. 2014;9(10):e110111.

10. Al-Khatib SM, Allen LaPointe NM, Chatterjee R, et al. Rate- and rhythm-control therapies in patients with atrial fibrillation: a systematic review. Ann Intern Med. 2014;160(11):760-73.

11. Oldgren J, Alings M, Darius H, et al. Risks for stroke, bleeding, and death in patients with atrial fibrillation receiving dabigatran or warfarin in relation to the $\mathrm{CHADS}_{2}$ score: a subgroup analysis of the RE-LY trial. Annals of internal medicine. 2011;155(10):6607, W204.

12. Dzeshka M, Lane D, Lip G. Stroke and Bleeding Risk in Atrial Fibrillation: Navigating the Alphabet Soup of Risk-Score Acronyms $\left(\mathrm{CHADS}_{2}, \mathrm{CHA}_{2} \mathrm{DS}_{2}\right.$-VASc, $\mathrm{R}_{2} \mathrm{CHADS}_{2}$, HAS-BLED, ATRIA, and More). Clinical Cardiology. 2014;37(10):634-644.

13. Hrynkiewicz-Szymanska A, Dluzniewski M, Platek AE, et al. Association of the CHADS2 and CHA 2DS 2-VASc scores with left atrial enlargement: a prospective cohort study of unselected atrial fibrillation patients. J Thromb Thrombolysis. 2014.

14. Albertsen I, Rasmussen L, Overvad T, Graungaard T, Larsen T, Lip G. Risk of Stroke or Systemic Embolism in Atrial Fibrillation Patients Treated With Warfarin A Systematic Review and Metaanalysis. Stroke. 2013;44(5):1329-1336.

15. Senoo K, Lip GY. Comparative efficacy and safety of the non-vitamin $\mathrm{k}$ antagonist oral anticoagulants for patients with nonvalvular atrial fibrillation. Semin Thromb Hemost. 2015;41(2):146-53.

16. Dentali F, Botto GL, Gianni M, Ambrosino P, Di Minno MN. Efficacy and safety of direct oral anticoagulants in patients undergoing cardioversion for atrial fibrillation: A systematic review and meta-analysis of the literature. Int J Cardiol. 2015;185:72-77.

17. Basili S, Raparelli V, Proietti M, Napoleone L, Ferroni P, Franconi F. Old And New Oral Anticoagulants In Management Of Atrial Fibrillation: A Double-Edged Sword For Women. Curr Vasc Pharmacol. 2015.

18. Cope S, Clemens A, Hammès F, Noack H, Jansen JP. Critical appraisal of network meta-analyses evaluating the efficacy and safety of new oral anticoagulants in atrial fibrillation stroke prevention trials. Value Health. 2015;18(2):234-49.

19. Claridge SB, Kanaganayagam GS, Kotecha T. Atrial fibrillation guidelines. Don't forget HASBLED score. BMJ. 2011;342:d3205.

20. Scowcroft AC, Lee S, Mant J. Thromboprophylaxis of elderly patients with AF in the UK: an analysis using the General Practice Research Database (GPRD) 2000-2009. Heart. 2013;99(2):12732 .
21. Stępińska J, Czerwińska K, Witkowski A, et al. Risk factors for bleeding complications in patients undergoing transcatheter aortic valve implantation (TAVI). Cardiol J. 2013;20(2):125-33.

22. Chiang C-EE, Naditch-Brûlé L, Murin J, et al. Distribution and risk profile of paroxysmal, persistent, and permanent atrial fibrillation in routine clinical practice: insight from the real-life global survey evaluating patients with atrial fibrillation international registry. Circ Arrhythm Electrophysiol. 2012;5(4):632-9.

23. Chan N, Paikin J, Hirsh J, Lauw M, Eikelboom J, Ginsberg J. New oral anticoagulants for stroke prevention in atrial fibrillation: impact of study design, double counting and unexpected findings on interpretation of study results and conclusions. Thrombosis and Haemostasis. 2014;111(5):798807.

24. Ahmad Y, Lip GY, Apostolakis S. New oral anticoagulants for stroke prevention in atrial fibrillation: impact of gender, heart failure, diabetes mellitus and paroxysmal atrial fibrillation. Expert Rev Cardiovasc Ther. 2012;10(12):1471-80.

25. Chugh SS, Roth GA, Gillum RF, Mensah GA. Global burden of atrial fibrillation in developed and developing nations. Glob Heart. 2014;9(1):113-9.

26. Alonso A, Krijthe BP, Aspelund T, et al. Simple risk model predicts incidence of atrial fibrillation in a racially and geographically diverse population: the CHARGE-AF consortium. J Am Heart Assoc. 2013;2(2):e000102.

27. Healey JS, Connolly SJ, Gold MR, et al. Subclinical atrial fibrillation and the risk of stroke. N Engl J Med. 2012;366(2):120-9.

28. Wachter R, Stahrenberg R, Gröschel K. Subclinical atrial fibrillation: how hard should we look? Heart. 2013;99(3):151-3.

29. Yoneyama K, Harada T, Ito H, et al. Subclinical atrial fibrillation preceding cardioembolic stroke in a patient with systolic heart failure. Int J Cardiol. 2014;176(3):1036-8.

30. Benezet-Mazuecos J, Rubio JM, Cortés M, et al. Silent ischaemic brain lesions related to atrial high rate episodes in patients with cardiac implantable electronic devices. Europace. 2015;17(3):364-9.

31. Hohnloser SH, Kuck KH, Lilienthal J. Rhythm or rate control in atrial fibrillation--Pharmacological Intervention in Atrial Fibrillation (PIAF): a randomised trial. Lancet. 2000;356(9244):1789-94.

32. Carlsson J, Miketic S, Windeler J, et al. Randomized trial of ratecontrol versus rhythm-control in persistent atrial fibrillation: the Strategies of Treatment of Atrial Fibrillation (STAF) study. J Am Coll Cardiol. 2003;41(10):1690-6.

33. Wyse DG, Waldo AL, DiMarco JP, et al. A comparison of rate control and rhythm control in patients with atrial fibrillation. $\mathrm{N}$ Engl J Med. 2002;347(23):1825-33.

34. Van Gelder IC, Van Veldhuisen DJ, Crijns HJ, et al. RAte Control Efficacy in permanent atrial fibrillation: a comparison between lenient versus strict rate control in patients with and without heart failure. Background, aims, and design of RACE II. Am Heart J. 2006;152(3):420-6.

35. Freudenberger RS, Wilson AC, Kostis JB. Comparison of rate versus rhythm control for atrial fibrillation in patients with left ventricular dysfunction (from the AFFIRM Study). Am J Cardiol. 2007;100(2):247-52. 
36. Van Gelder IC, Groenveld HF, Crijns HJ, et al. Lenient versus strict rate control in patients with atrial fibrillation. N Engl J Med. 2010;362(15):1363-73.

37. Smit MD, Crijns HJ, Tijssen JG, et al. Effect of lenient versus strict rate control on cardiac remodeling in patients with atrial fibrillation data of the RACE II (RAte Control Efficacy in permanent atrial fibrillation II) study. J Am Coll Cardiol. 2011;58(9):942-9.

38. Weipert KF, Erkapic D, Schmitt J. [Rate and rhythm control in atrial fibrillation: Pharmacological approaches]. Herz. 2015;40(1):16-24.

39. Rolf S, Kornej J, Dagres N, Hindricks G. What can rhythm control therapy contribute to prognosis in atrial fibrillation? Heart. 2015.

40. Heidbuchel H, Verhamme P, Alings M, et al. European Heart Rhythm Association Practical Guide on the use of new oral anticoagulants in patients with non-valvular atrial fibrillation. Europace. 2013;15(5):625-651.

41. Ruff CT, Giugliano RP, Braunwald E, et al. Comparison of the efficacy and safety of new oral anticoagulants with warfarin in pa- tients with atrial fibrillation: a meta-analysis of randomised trials. Lancet. 2014;383(9921):955-62.

42. Wallentin L, Lopes RD, Hanna M, et al. Efficacy and safety of apixaban compared with warfarin at different levels of predicted international normalized ratio control for stroke prevention in atrial fibrillation. Circulation. 2013;127(22):2166-76.

43. Connolly SJ, Ezekowitz MD, Yusuf S, et al. Dabigatran versus warfarin in patients with atrial fibrillation. N Engl J Med. 2009;361(12):1139-51.

44. Patel MR, Mahaffey KW, Garg J, et al. Rivaroxaban versus warfarin in nonvalvular atrial fibrillation. N Engl J Med. 2011;365(10):883-91.

45. Freemantle N, Lafuente-Lafuente C, Mitchell S, Eckert L, Reynolds M. Mixed treatment comparison of dronedarone, amiodarone, sotalol, flecainide, and propafenone, for the management of atrial fibrillation. Europace. 2011;13(3):329-45.

46. Lafuente-Lafuente C, Valembois L, Bergmann J-FF, Belmin J. Antiarrhythmics for maintaining sinus rhythm after cardioversion of atrial fibrillation. Cochrane Database Syst Rev. 2015;3:CD005049. 